\title{
Energía de Ionización simple en la Soldadura con Electrodo Revestido
}

\author{
(Simple Ionization Energy in Coated Electrode Welding)
}

\author{
Alejandro García Rodríguez ${ }^{1}$ \\ ${ }^{1}$ Universidad Central "Marta Abreu” de las Villas, Centro de Investigaciones de Soldadura (CIS) /Ministerio de Educación Superior \\ (MES), Santa Clara, Villa Clara,Cuba,alejo2@uclv.edu.cu
}

\begin{abstract}
Resumen
El objetivo del presente trabajo es presentar, a la comunidad cientifica internacional, la concepción de un método de estimación de la energía de ionización simple, indispensable para el establecimiento del plasma térmico necesario para realizar el proceso de soldadura con electrodo revestido. A partir de la síntesis y el análisis de resultados teóricos y experimentales establecidos en la literatura especializada, fue deducido un método de estimación de la energía invertida en el proceso de ionización de determinado porcentaje de los elementos disociados que componen el gas heterogéneo (resultante de la descomposición de la masa de revestimiento) en la unidad de tiempo, en función de la temperatura. La eficacia de la función eléctrica del revestimiento posibilita el desarrollo de las funciones metalúrgicas y operativas del consumible, dependiendo de las propiedades físico-químicas de los materiales del revestimiento y sus concentraciones relativas. La determinación de las proporciones exactas de los componentes de las mezclas que integran los revestimientos de los electrodos revestidos, constituye un importante reto tecnológico para los fabricantes, dadas las diferencias de composición química de las materias primas comercializadas y la necesidad de minimizar, integralmente, la relación costo-beneficio del producto. Una adecuada estabilidad eléctrica del proceso es imprescindible para obtener una óptima calidad de la unión soldada.
\end{abstract}

Palabras-Clave: Estimación, Potencial de ionización, Composición del revestimiento, Función eléctrica, Grado de ionización.

\begin{abstract}
The objective of the present work is to present to the international scientific community the conception of an estimation method of the simple ionization energy, indispensable for the establishment of the thermal plasma needed for covered electrode welding. Starting from the synthesis and the analysis of experimental and theoretical results established in the specialized literature, it was deduced a method for estimating the invested energy in the ionization process of determinate percentage of the disassociated elements component of the heterogeneous gas (resulting of the decomposition of the coating mass), in the time unit, in function of the temperature. The effectiveness of the coating electric function make possible the development of the metallurgic and operatives functions of the electrode, depending on the physical and chemical properties of the coating materials and their relative concentrations. The determination of the exact proportions of the components of the mixtures integrating the covered electrode coatings constitutes an important technologic challenge for manufacturers, because of the chemical composition differences of the raw material and the necessity of minimization of the product cost benefit relation. An appropriate electric stability of the process is essential for obtaining an optimal quality of the welded union.
\end{abstract}

Key-Words: Estimation, Potential of ionization, Coating composition, Electric function, Grade of ionization.

\section{Introducción}

En 1802, V.V. Petrov descubrió la descarga de arco en atmósfera abierta. El "arco de Petrov", tal y como se conoce en la literatura soviética [1] constituyó un hecho de gran importancia en la historia de la electrónica. Recordemos que la primera fuente de luz eléctrica técnicamente aceptable (bujía de Yáblotchkov) utiliza un arco con una configuración especial de los electrodos que permite un mantenimiento prolongado de la descarga; mientras que los métodos de corte y soldadura

(Recebido em 09/08/2012; Texto final em 17/09/2012). de metales inventados por N.G. Slaviánov y N.I.Benardos se basan en la utilización de la alta temperatura del arco eléctrico en el aire. En 1904, basado en semejantes principios, el célebre ciudadano sueco Oscar Kjellberg, fundador de la aún vigente y exitosa empresa ESAB, patentó el proceso de soldadura manual con electrodo revestido, comúnmente conocido en idioma inglés como Manual Metal Arc (MMA), también Shielded Metal Arc Welding (SMAW) o, en el Reino Unido, Stick Electrode Welding. Desde entonces este versátil proceso tecnológico se mantiene aún insustituible frente a los procesos automáticos de soldadura, en diversas aplicaciones industriales a nivel mundial. Según estudios [2], se considera que para el año 2013 aproximadamente el $40 \%$ del consumo de metal por soldadura a nivel mundial se seguirá realizando a partir del empleo de electrodos. 
En el proceso de soldadura con electrodo revestido, clasificado como proceso de electrodo consumible (cátodo frío), el mecanismo de conducción a través de la región de caída del cátodo, es el de emisión de electrones en una superficie, debido a la aplicación de un campo eléctrico externo [3]. La densidad de corriente electrónica depende de la intensidad de campo, la función de trabajo de la superficie metálica y la Energía de Fermi para el material del electrodo (cátodo). Ha sido demostrado [4] que aún cuando la función de trabajo de la superficie metálica (trabajo de extracción de los electrones) cae por debajo de su rango normal de $3,5 \mathrm{a} 4,5 \mathrm{eV}$, a valores de $2 \mathrm{eV}$, debido a la influencia de las capas de óxido, la emisión de campo sólo puede ocurrir para densidades de corriente mayores que $10^{11} \mathrm{~A} \cdot \mathrm{m}^{-2}$.

Existen evidencias sobre la importancia de las capas de óxido del cátodo en ciertos arcos, incluyendo algunos usados en soldadura [5]. Finas capas aislantes de óxido en la superficie del cátodo pueden cargarse por la incidencia de iones positivos. Para películas de óxido de espesores menores de $5 \times 10^{-9} \mathrm{~m}$, el campo eléctrico a través de ella debe excederse sobre $2 \times 10^{9} \mathrm{~V} \cdot \mathrm{m}^{-1}$ para la emisión de electrones por Efecto Túnel. Si la película de óxido tiene un espesor menor que $10^{-8} \mathrm{~m}$ y es cargada por iones positivos, de la caída del cátodo ante el campo eléctrico se producen canales intermitentes eléctricamente conductivos. Se ha sugerido [6] que el campo eléctrico en la fase gaseosa del cátodo pudiera solo necesitar $10^{5} \mathrm{~V} . \mathrm{m}^{-1}$ para desencadenar la emisión.

El uso de revestimientos no metálicos, que produce finas capas superficiales de óxido de materiales semiconductores a altas temperaturas, posibilita la soldadura con electrodo negativo en corriente directa y en corriente alterna; aunque la polaridad cambie, el metal se transfiere en un mismo sentido (del electrodo a la pieza). Esto se debe fundamentalmente a que esas capas dan continuamente nuevos sitios de emisión cercanos a la punta del electrodo [4].

La composición de los revestimientos de los electrodos se constituye de una mezcla de sustancias orgánicas y minerales. En la fabricación de la pasta para el revestimiento intervienen óxidos naturales, como óxido de hierro (polvo de hierro), ilmenita (50 por ciento óxido férrico y 50 por ciento óxido de titanio), sílice (óxido de silicio), silicatos naturales (caolín, talco, mica, feldespato, etc), productos volátiles (celulosa, serrín, etc), fundentes (fluorita), concentrados (carbonatos, rutilo), ferroaleaciones (FeMn, FeSi, FeTi) y aglomerantes (silicato sódico, silicato potásico) [7]. La composición, el origen, la dosificación y el tratamiento de las sustancias utilizadas en la fabricación del revestimiento de los electrodos desempeñan un papel fundamental en la soldadura SMAW. Las fórmulas de preparación de la mezcla de los diversos tipos de revestimientos constituyen un secreto vital para los fabricantes, parte del know-how de las transnacionales productoras de todo tipo de electrodos revestidos.

La energía producida por efecto Joule al paso de la corriente de emisión de electrones, en presencia del campo eléctrico, constituye la fuente energética que produce la descomposición del revestimiento, vaporiza los metales y sostiene las reacciones químicas de disociación de las sustancias en átomos y moléculas que componen el gas protector del arco. En esta etapa, los gases están constituidos por átomos y moléculas neutros que no conducen la corriente eléctrica puesto que aún no han sido ionizados y no existen portadores de carga.

Según la literatura, el arco eléctrico es una forma de descarga eléctrica en un gas o en un vapor en el que existe una caída catódica de potencial del orden del potencial de ionización o de excitación del gas [1]. El paso de un gas al estado de plasma está relacionado con varios procesos de interacción entre las partículas, producidos a causa de choques entre partículas o por interacciones con determinadas radiaciones. El Proceso de Ionización, es decir, la separación de un electrón de un átomo o molécula que genera un ión de carga positiva y el Proceso de Recombinación, que es el proceso inverso, son los más importantes. La ionización es un proceso que tiene umbral, o sea, que para que tenga lugar se necesita que la energía de las partículas que chocan, o del cuanto de luz, debe ser mayor que la umbral, la cual depende de la resistencia del átomo. Este umbral se denomina energía de ionización. Para que se produzca la recombinación debe cumplirse la condición inversa, es decir que el átomo que se forma tiene que desprenderse de la energía sobrante, de lo contrario se vuelve a deshacer inmediatamente [8].

La reacción de ionización es endotérmica, mientras que la de recombinación es exotérmica y contribuye a elevar la temperatura del arco. La cantidad de energía transportada a través del arco se relaciona con el potencial de ionización de las especies químicas ionizadas. Dado que el gas producido por la descomposición de las diversas sustancias componentes del revestimiento presenta moléculas y átomos de diferentes potenciales de ionización, se añaden sustancias de bajo potencial de ionización (Tabla 1) como el sodio $(\mathrm{Na})$ y el potasio $(\mathrm{K})$ que facilitan el inicio del proceso de cebado del arco; sin embargo, un exceso de sustancias de bajo potencial de ionización respecto a aquellas de mayor potencial, limita la capacidad de transporte de calor a través del arco, afectándose la eficiencia del proceso.

De acuerdo con lo escrito anteriormente, para ionizar las moléculas y/o átomos presentes en un gas, hay que realizar el trabajo de ionización $\left(A_{i}\right)$ contra las fuerzas de atracción entre el electrón que se arranca y el residuo atómico, es decir, las demás partículas del átomo. La magnitud $A_{i}$ depende del estado energético del electrón que se arranca en el átomo o molécula del gas dado, mientras que se denomina potencial de ionización $\varphi_{i}$, la diferencia de potencial del campo eléctrico acelerador que debe recorrer la partícula cargada para acumular una energía igual a la de ionización (Ecuación 1) [9]:

$$
\varphi_{i}=A_{i} / e
$$

Donde $\varphi_{i}$ es el potencial de ionización, $A_{i}$ es la energía de ionización y e es la magnitud absoluta de la carga de un electrón. 
Tabla 1: Potenciales de ionización $\left(\varphi_{i}\right)$ de algunas sustancias en Volts

\begin{tabular}{|l|l|l|l|}
\hline \multirow{2}{*}{ Elemento } & \multicolumn{3}{|l|}{ Estado de ionización* } \\
\cline { 2 - 4 } & I (Simple) & II & III \\
\hline Potasio & 4,339 & 31,81 & 46 \\
\hline Sodio & 5,138 & 47,29 & 71,65 \\
\hline Calcio & 6,11 & 11,87 & 51,21 \\
\hline Titanio & 6,83 & 13,63 & 28,14 \\
\hline Hierro & 7,90 & 16,18 & 30,64 \\
\hline Silicio & 8,149 & 16,34 & 33,46 \\
\hline Carbono & 11,264 & 24,376 & 47,864 \\
\hline Hidrógeno & 13,595 & & \\
\hline Oxígeno & 13,614 & 35,146 & 54,934 \\
\hline Nitrógeno & 14,54 & 29,605 & 47,426 \\
\hline Argón & 15,755 & 27,62 & 40,90 \\
\hline
\end{tabular}

*El número romano indica el número de electrones que se pierden en el estado de ionización (datos tomados del libro "The Physics of Welding”. J.F. Lancaster [4],pág 15).

Nótese que el potencial de ionización de los vapores metálicos es mucho menor que el potencial de ionización de gases como el oxígeno, el nitrógeno y el argón. De esta manera, en una mezcla de vapores de metal y gas a $6000 \mathrm{~K}$, el vapor metálico estará altamente ionizado mientras que el gas estará sustancialmente des-ionizado. Consecuentemente, se ha especulado que en los arcos de soldadura la conductividad eléctrica de la columna del arco pudiera deberse a la ionización de pequeñas cantidades vapores de metal derivados de los electrodos. Mediciones de temperatura sugieren que esto es cierto para arcos con hierro o electrodos de acero [4].

Debido a la magnitud de las temperaturas (6000 K a 20000 $\mathrm{K}$ ) de los vapores del arco de soldadura, la medición directa de sus propiedades se hace en extremo difícil sino imposible. Por este motivo a menudo se hace necesario el uso de leyes físicas conocidas para obtener, mediante cálculos, aproximaciones a las propiedades requeridas [10]. Para lograr esto, se asume que el gas es eléctricamente neutro, y que se encuentra en equilibrio térmico, o sea que la distribución de energía de las partículas es normal y que la temperatura de los electrones es igual a la de las partículas neutras. Estrictamente hablando, un gas en que existe un proceso de difusión tal como el flujo de calor o de corriente eléctrica no puede estar en equilibrio, sin embargo, a las temperaturas del arco de soldadura con electrodo revestido, en que el grado de ionización es pequeño (gas denso y débilmente ionizado), constituyen una buena aproximación las condiciones planteadas anteriormente. Un gas ionizado, eléctricamente neutro, es conocido como plasma [4], mientras que un gas denso y débilmente ionizado, a esta temperatura, puede ser considerado en equilibrio térmico y algunas de sus propiedades son definibles en base a las leyes y principios de la termodinámica [8].

La ionización debida a los choques entre moléculas neutrales (ionización térmica) juega un papel notable en este tipo de descargas de arco a alta presión. La teoría de la ionización térmica fue desarrollada por el científico indio Saha [1], quien definió una ecuación termodinámica que permite el cálculo del grado de ionización de distintas sustancias en función de la temperatura, entre otros parámetros.

$\frac{\alpha^{2}}{1-\alpha^{2}}=2,4 \cdot 10^{-4} \cdot \frac{T^{5 / 2} \cdot \exp \left\{-\frac{11600 \cdot U_{i}}{T}\right\}}{p} \approx \alpha^{2}$

Donde $U_{i}$ es el potencial de ionización ( $\varphi_{i}$ en Ecuación 1$)$ y está expresado en volts como en la Tabla $1, p$ es la presión en mm $\mathrm{Hg}$, y $\alpha$ es la relación entre el número de moléculas ionizadas y su número total (grado de ionización). Cuando $\alpha^{2}$ es mucho menor que 1 , el término $\alpha^{2} /\left(1-\alpha^{2}\right)$ se puede sustituir por $\alpha^{2}$.

De la Ecuación 2 obsérvese que para las sustancias de mayores valores de potencial de ionización, a temperatura y presión constantes, se obtendrán menores valores $\alpha$, mientras que, consistentemente, de la Figura 1, se observa que para elementos de más bajo potencial de ionización (Tabla 1) se obtienen grados de ionización superiores a más bajas temperaturas, mientras que los elementos de mayor potencial de ionización como el Oxígeno y el Nitrógeno necesitan temperaturas mucho más altas para obtener los mismos valores de $\alpha$.

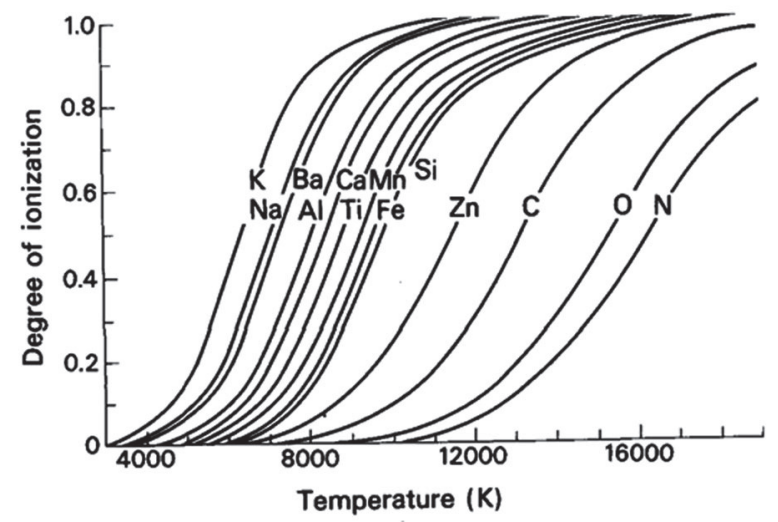

Figura 1. Grado de ionización (escala logarítmica) como función de la temperatura para determinado número de elementos (tomado del libro "The Physics of Welding”. J.F. Lancaster [4], pág 138).

En el gas heterogéneo presente en el arco de soldadura con electrodo revestido existen átomos de diferente naturaleza, con diferentes potenciales de ionización, sometidos a las condiciones de temperatura y presión impuestas por el régimen de trabajo del proceso, por lo que de acuerdo con los resultados expuestos en el presente trabajo, se hace evidente que los primeros elementos químicos en ionizarse serán los de menor potencial de ionización, contribuyendo a un aumento de la temperatura del arco que permita la ionización de otros elementos de mayor potencial de ionización, aportando la energía indispensable para lograr el establecimiento de un plasma estable, capaz de recuperarse rápidamente de las afectaciones producidas por los eventos desestabilizadores del arco y del proceso, típicos de las condiciones prácticas naturales de su ejecución. 
Encontrar las proporciones exactas de los componentes del revestimiento para maximizar la eficiencia eléctrica del proceso, considerando la necesidad de mantener una óptima relación costo-beneficio en los procesos de diseño, fabricación y comercialización de los electrodos revestidos, es una tarea constante para los fabricantes de todo el mundo. El objetivo del presente trabajo es presentar, a la comunidad científica internacional, la concepción de un método de estimación de la energía de ionización simple, indispensable para el establecimiento del plasma térmico necesario para realizar el proceso de soldadura con electrodo revestido.

\section{Desarrollo}

En el caso de la soldadura con electrodo revestido debe tenerse en cuenta que el intenso calor del arco funde el metal del núcleo del electrodo y a su vez su revestimiento, por lo que el gas que compone el plasma estará compuesto por diversas sustancias, entre las que los vapores del metal del núcleo del electrodo juegan un papel fundamental. Si el proceso es suficientemente estable, es decir, que la estabilidad del aporte energético permite que se obtenga una calidad del cordón de soldadura similar desde el inicio hasta el final, producto de la fusión de un electrodo completo; es posible suponer que la velocidad de soldadura y la tasa de fusión del electrodo se mantienen constantes y, conociendo el peso del electrodo y el tiempo total de soldadura, calcular qué porción del electrodo se consume en la unidad de tiempo.

Conociendo que la facilidad de cebado y mantenimiento del arco depende fundamentalmente de la temperatura alcanzada en el arco, del potencial de ionización, del grado de ionización alcanzado por las sustancias en estas condiciones (Figura 1) y las características operativas del proceso, es posible calcular la cantidad de gramos de revestimiento consumidos en cada segundo que, multiplicada por la fracción de cada óxido y por la constante gravimétrica del elemento oxidado, se corresponde con el peso de sus átomos en esa cantidad. Teniendo en cuenta el peso de $1 \mathrm{~mol}$ del elemento, cuya cantidad se quiere determinar dentro del óxido, se puede obtener la fracción molar que nos permite determinar la cantidad de electrones e iones que pueden formarse al producirse la ionización del elemento, que multiplicada por su grado de ionización a determinada temperatura, constituye la cantidad de partículas cargadas generadas en el gas.

\subsection{Cantidad de Revestimiento Consumido}

Para la determinación de la cantidad de masa de revestimiento consumido en cada segundo $\left(M_{S}\right)$, es necesario determinar el tiempo promedio $\left(T_{F}\right)$ que demoran en consumirse varios electrodos en segundo y la masa promedio de revestimiento del electrodo en cuestión $\left(M_{E}\right)$, obtenida a partir de un preciso pesaje del revestimiento de una muestra de electrodos suficientemente significativa desde el punto de vista estadístico (Ecuación 3).

$$
M_{S}=\frac{M_{E}}{T_{F}}
$$

La masa considerada estará compuesta por todos los componentes del revestimiento, en las proporciones determinadas por la fórmula empleada por el fabricante.

\subsection{Fracción Molar}

Asumida una correcta homogeneidad del revestimiento, las proporciones de los elementos químicos en la masa $\mathrm{M}_{\mathrm{S}}$ de revestimiento se mantendrán constantes en toda fracción de masa de revestimiento a lo largo del electrodo. Como cada óxido responde a la forma $X_{a} O_{b}$, donde $X$ es un determinado elemento químico ( $\mathrm{Si}, \mathrm{Fe}, \mathrm{Mn}$, etc.); y $\mathrm{O}$ es el oxígeno, para determinar el porciento en peso de cada elemento acompañante del oxígeno, es necesario tener en cuenta la relación extequiométrica de cada óxido particular, respecto al elemento que acompaña al oxígeno $\left(R_{E i}\right)$, según la Ecuación 4.

$R_{E_{i}}=\frac{P_{E_{i}}}{P_{O x i}}$

Donde $P_{E i}$ es el peso atómico de $1 \mathrm{~mol}$ del elemento y $P_{O x i}$, el peso atómico total de $1 \mathrm{~mol}$ del óxido.

Esta relación se mantiene constante para cada compuesto, por lo que para determinar el porciento en peso del elemento consumido en cada segundo, se necesita calcular el peso del óxido y multiplicarlo por $R_{E i}$, (Ecuación 5)

$$
F_{M i}=\frac{\left(\% E_{i} /{ }_{100}\right) \cdot R_{E i} \cdot M_{S}}{P_{E i}}
$$

Donde $F_{M i}$ es la relación entre el peso del elemento contenido en la cantidad de masa de revestimiento consumida cada segundo y el peso atómico de $1 \mathrm{~mol}$ del elemento $E i$ (fracción molar); $\% E i$, el porciento en peso determinado por análisis químico en $100 \mathrm{~g}$ de revestimiento; $R_{E i}$ la relación expresada en la Ecuación 4; $M_{S}$ se define en la Ecuación 3, y $P_{E i}$ es el peso atómico de 1 mol del elemento en cuestión.

\subsection{Cantidad de Átomos por Mol}

Para estimar la cantidad de átomos por elemento $C_{A i}$ (Ecuación 6), presentes en la mezcla, basta con multiplicar el valor obtenido en la Ecuación $5\left(F_{M i}\right)$, de cada elemento, por el número de Avogadro $\left(N_{A}\right)$, cuyo significado es aceptado como el número de partículas por mol de cualquier sustancia.

$$
C_{A i}=N_{A} \cdot F_{M i}
$$

Siendo $N_{A}=6.02310^{23}$ partículas / mol y $F_{M i}$ la fracción molar del elemento calculada en la Ecuación 5.

\subsection{Cantidad de Electrones}

Conociendo el potencial de ionización de cada elemento (Tabla 1) puede obtenerse el grado de ionización $(\alpha)$ de las 
sustancias componentes a diferentes temperaturas (Ecuación 2, Figura 1). Para el caso de la soldadura con electrodo revestido con núcleo de hierro, vale asumir $6000 \mathrm{~K}$ [4]. El grado de ionización indica qué porciento de la cantidad total de partículas de cada elemento, realmente se ioniza a determinada temperatura. Por cada átomo ionizado se libera un electrón, formándose un ión cargado positivamente.

Para calcular la cantidad de electrones obtenidos a partir de la ionización de la cantidad de átomos de cada elemento $E i$ (Ecuación 7), se debe multiplicar el grado de ionización $\left(\alpha_{i}\right)$ por la cantidad de partículas calculadas anteriormente $\left(C_{A i}\right)$ de la Ecuación 6.

$$
n e E_{i}=\alpha_{i} \cdot C_{A i}
$$

\subsection{Energía de ionización por elemento y total}

Para calcular la energía necesaria para ionizar una cierta cantidad de partículas de determinado elemento químico $E_{i}$, se puede multiplicar la carga del electrón $\left(\mathrm{e}=1,60210^{-19} \mathrm{C}\right)$ por el potencial de ionización $\left(\varphi_{i}\right)$ del átomo del elemento en cuestión (Ecuación 1, Tabla 1), por la cantidad de electrones calculada en la Ecuación 7, como se expresa en la Ecuación 8.

$$
E E_{i}=1,602.10^{-19} \cdot \varphi i \cdot n e E_{i}
$$

Donde $E E_{i}$, es la energía consumida por las reacciones de ionización de una fracción de los átomos del elemento $E$, contenidos en el gas disociado.

Finalmente, una estimación de la energía total de ionización simple (EIT) de una parte de los elementos contenidos en la fracción de revestimiento consumido por unidad de tiempo, se puede calcular a partir de la Ecuación 9.

$$
E I T=\sum_{i=1}^{N} E E_{i}
$$

Donde $1 \leq i \leq N$ y $N$ es la cantidad de diferentes elementos presentes en la mezcla del revestimiento.

\section{Conclusiones}

1.El método planteado permite estimar la energía invertida en el proceso de ionización simple de cierto porcentaje de los elementos del gas heterogéneo resultante de la descomposición de determinada masa de revestimiento, en la unidad de tiempo, en función de la temperatura alcanzada en el proceso de soldadura manual con electrodo revestido.

2. La magnitud de la energía consumida en el proceso de ionización, hasta el umbral de establecimiento del plasma, depende, entre otros factores, de la naturaleza y dosificación de los componentes de bajo potencial de ionización respecto a los elementos de mayor potencial de ionización.

3. La minimización de la relación costo-beneficio pasa por un constante proceso de ajuste de las fórmulas de los revestimientos en función de los precios, cualidades $\mathrm{y}$ calidades de las materias primas y sus tecnologías de preparación, sin detrimento de la función eléctrica del revestimiento; para cuyo balance el método planteado puede ser de gran importancia.

4. Validar experimentalmente el grado de precisión en la estimación de la energía de ionización simple en los gases del proceso de soldadura manual con electrodos revestidos y su correlación con el comportamiento eléctrico del arco logrado con diferentes tipos de electrodos revestidos, puede ser un reto tecnológico interesante en el campo de la soldadura moderna y puede auxiliar el estudio del efecto del empleo de nuevos materiales constituyentes del revestimiento.

5. Del análisis desarrollado se desprende que el método planteado puede ser generalizado a otros procesos de soldadura por arco eléctrico, en caso de que en los regímenes de trabajo típicos de cada proceso sea posible asumir las condiciones especificadas en el trabajo y los resultados puedan ser corroborados experimentalmente, lo cual constituye un interesante tema de investigación.

\section{Agradecimientos}

Mis más sinceros agradecimientos a todos aquellos que de una forma $\mathrm{u}$ otra han colaborado con mi formación profesional tanto en el plano institucional como familiar y en especial, por su desinteresada colaboración, a los doctores Rafael Quintana Puchol y Carlos René Gómez Pérez del Centro de Investigaciones de Soldadura de la Universidad Central "Marta Abreu" de Las Villas.

\section{Referencias Bibliográficas}

[1] Gaponov. VI. Física Electrónica. Ciencia y Técnica. La Habana, Cuba. 1968.

[2] ESAB. Business and financial review: ESAB; 2006

[3] Fowler RH, Nordheim L. Electron emission in intense electric fields. Proc. Roy. Soc. 1928 \& 192; A119, A124: 173, 699.

[4] Lancaster JF. The physics of Welding. 2aed. Oxford: International Institute of Welding; 1986.

[5] Guile AE. Processes at arc cathode roots on non-refractory arc cathodes with relatively thick oxide films. Arc Physics and Weld Pool Behaviour. The welding Institute, London.1979.

[6] Guile AE. Studies of short electric arcs in transverse magnetic fields with application to arc welding. Welding in the world. 1970; 8(1):36.

[7] Ramírez J. Soldadura por arco manual con electrodos revestidos [Monografía en Internet]. Universidad Central de Venezuela: Facultad de Ingeniería, Escuela de Ingeniería; 2003 [Consultado en febrero 2008] http://www.monografías.com/ trabajos7/elecrev/elecrev.shtml?askjeeves.

[8] Frank-Kamenetski DA. El plasma, cuarto estado de la materia. Editorial MIR. Moscú.1970.

[9] Yavorski BM, Detlaf AA. Prontuario de Física. Ed MIR. Moscú.1983.

[10] Schellhase M. Der SchweiBlichtbogen-ein technologisches Wérkzeug. Berlín: Técnica de Berlín; 1985. 